\title{
Morphological Effects of Various Silica Nanostructures on the Mechanical Properties of Printed Parts in Digital Light Projection 3D Printing
}

Alamelu Suriya Subramanian ${ }^{a, b}$, Erwin Peng $^{c}$, Wei Cheng Lau ${ }^{b}$, Darren Chuen Wee Goh ${ }^{b}$,

Steven Pramono ${ }^{c}$, Deepa Sriramulu ${ }^{c}$, Yili Wu ${ }^{c}$, Hitoshi Kobayashi ${ }^{c}$, James Guo Sheng

$\mathrm{Moo}^{c, *}$, Pei-Chen $\mathrm{Su}^{a, b, *}$

a Singapore Centre for 3D Printing (SC3DP), Nanyang Technological University, 50 Nanyang Avenue, N3.1-B2C-03, Singapore 639798

b School of Mechanical and Aerospace Engineering, Nanyang Technological University, 50 Nanyang Ave, Block N3, Nanyang Ave, 639798

c Evonik (SEA) Pte. Ltd. Asia Research Hub, 21 Biopolis Road, \#01-35 Nucleos Tower A (South) Level 1M, Singapore 138567

Correspondence: peichensu@ntu.edu.sg (P.-C. S.); james.moo@evonik.com (J.G.S.M.) 


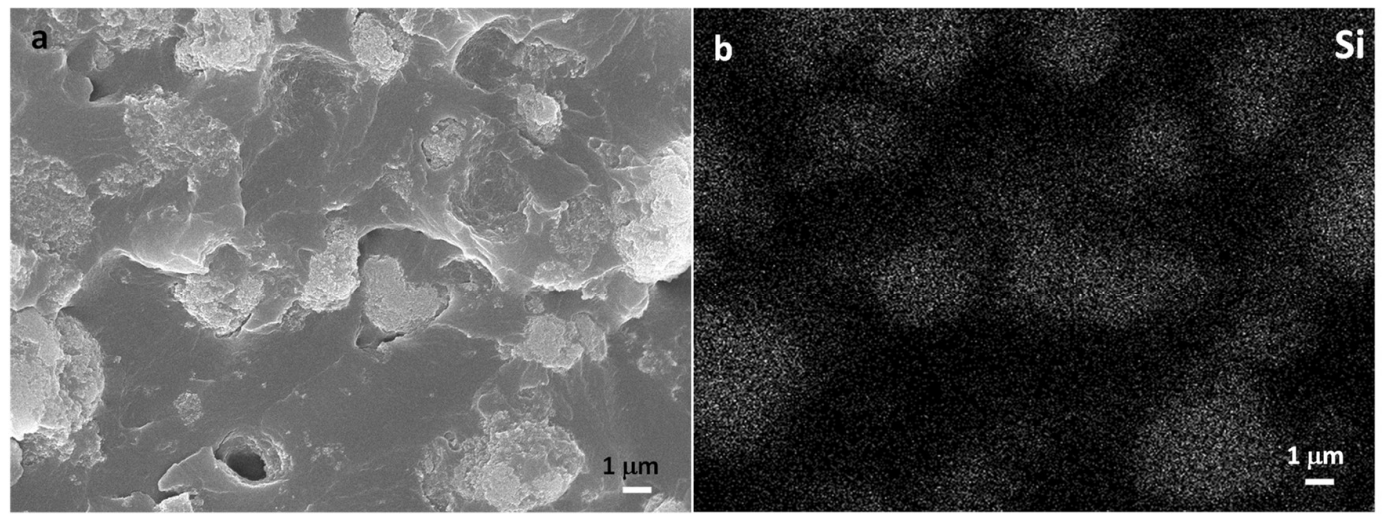

FL Tough + HEMA + 10 wt. \% PP1

Figure S1. (a) SEM image and (b) EDS analysis of precipitated silica PP1 in cured Tough + HEMA matrix.

a

Formlabs Elastic + HEMA

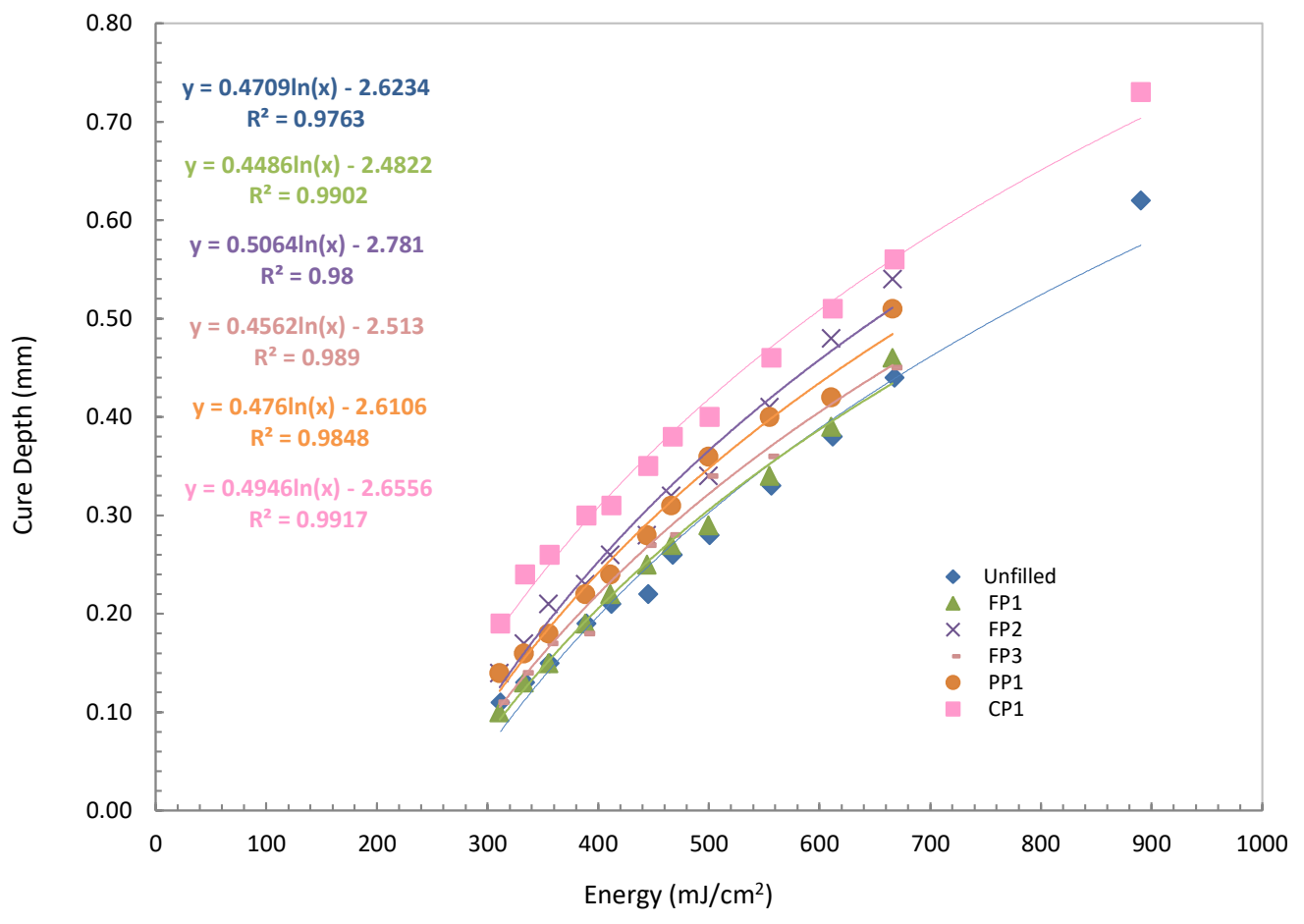




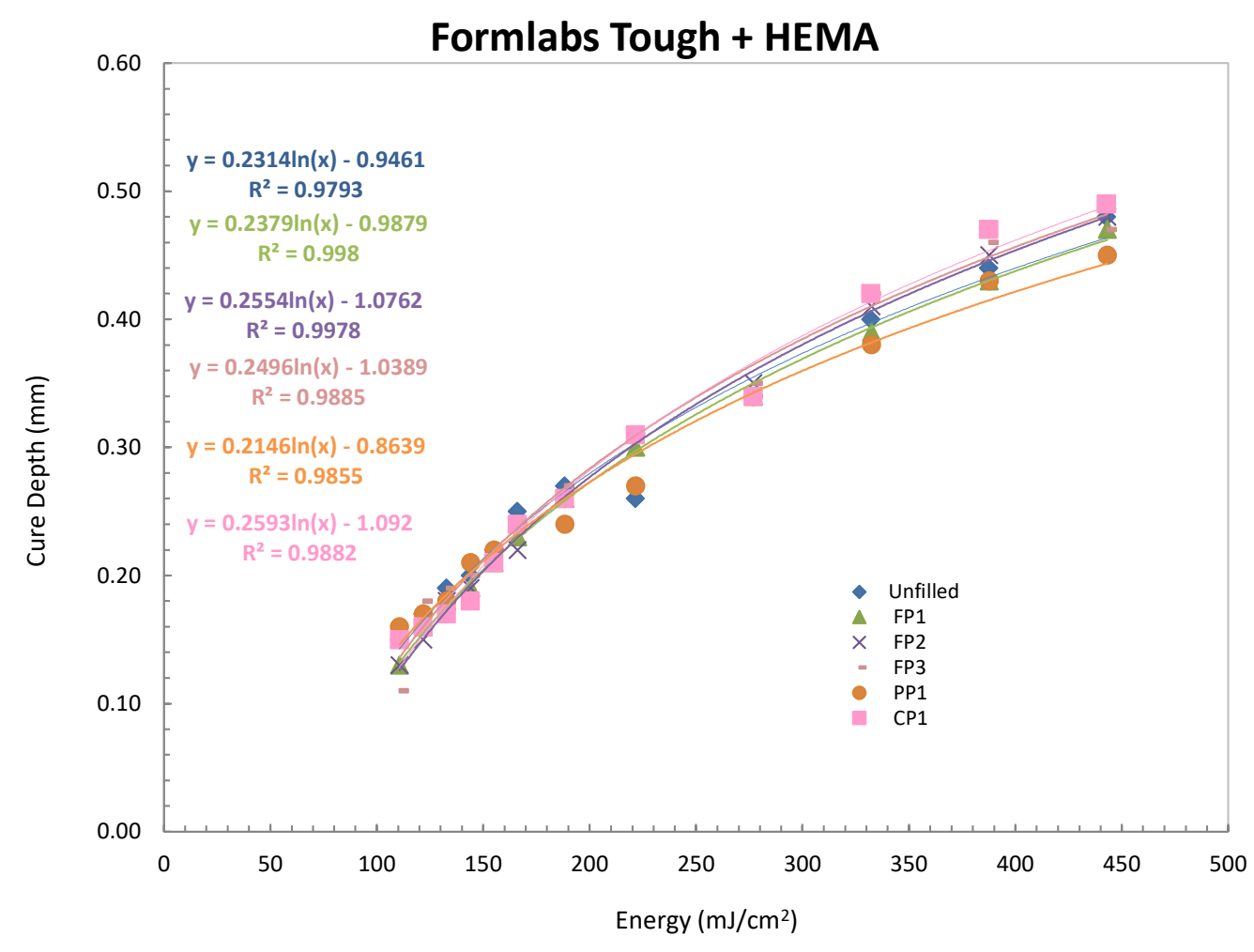

C

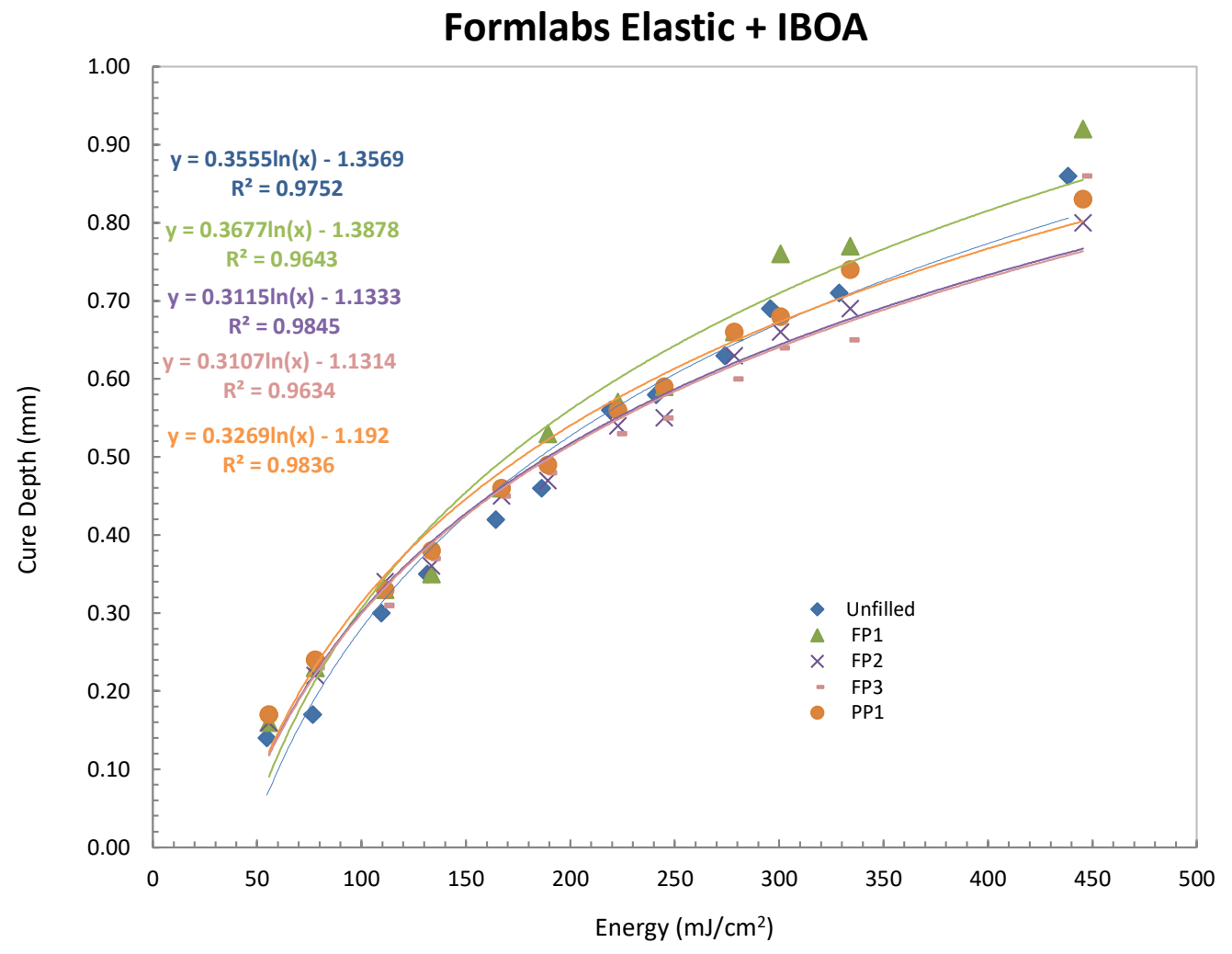




\section{Supporting Information}

d

\section{Formlabs Tough + IBOA}

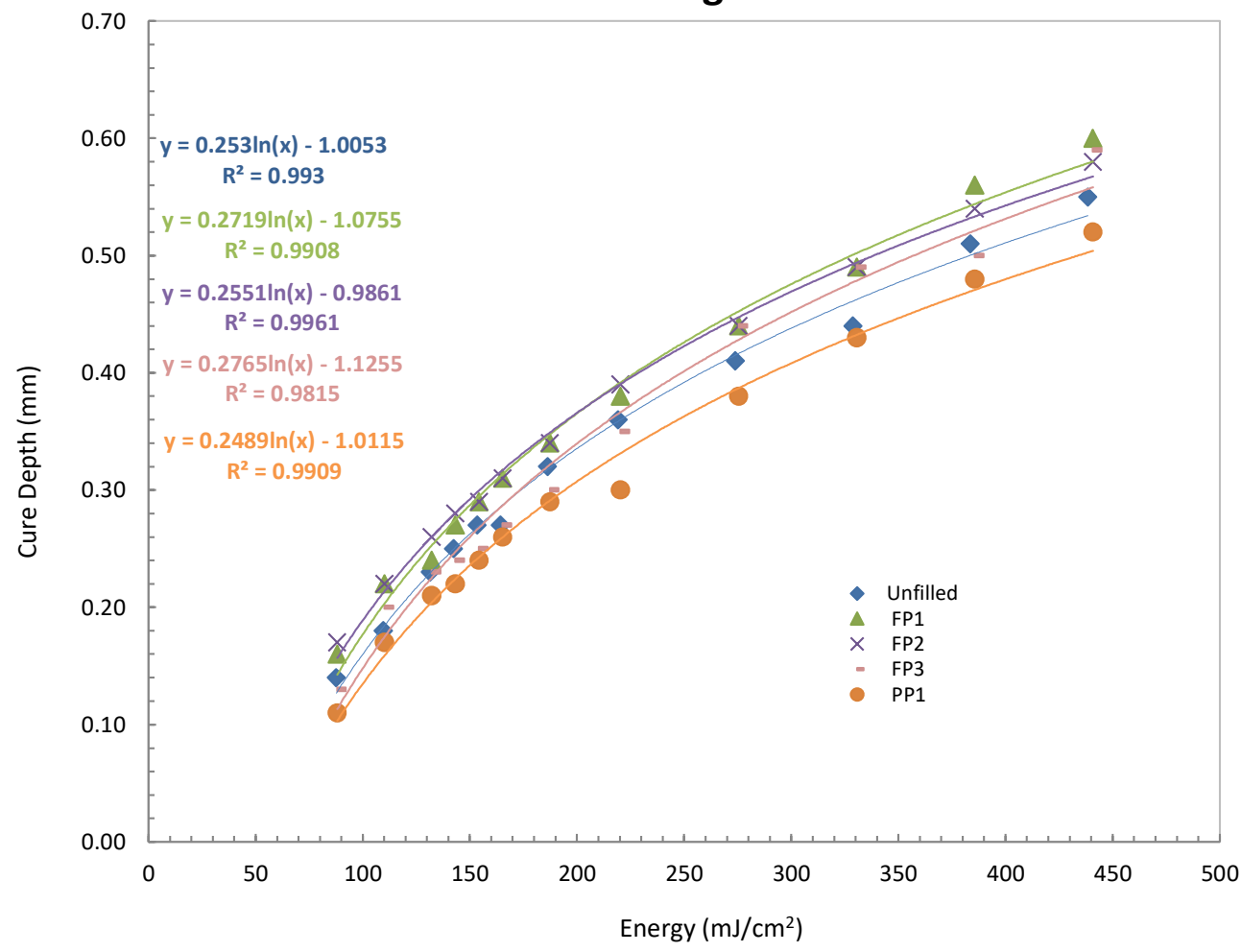

Figure S2. Cure depth curves for (a) Formlabs Elastic Resin with HEMA diluent, (b) Formlabs Tough Resin with HEMA diluent, (c) Formlabs Elastic Resin with IBOA diluent, and (d) Formlabs Tough Resin with IBOA diluent. 